\title{
Self-made adjustable external fixation device with progressive compression for the management of open fracture with bone defects of the distal hallux phalanx: a case report
}

\author{
Ying-Guo YANG \\ sihong county people's hospital \\ Da-Ke Tong \\ Changhai Hospital \\ peizhao Liu ( $\square$ lpzhao089@163.com ) \\ PLA wuhan General Hospital
}

\section{Research article}

Keywords: Open Fracture, Distal Phalanx of the Hallux, Compressive External Fixation Device, Bone Defect

Posted Date: February 11th, 2020

DOl: https://doi.org/10.21203/rs.2.23035/v1

License: (c) (1) This work is licensed under a Creative Commons Attribution 4.0 International License.

Read Full License 


\section{Abstract}

Background: Open fractures with bone defects of the distal phalanx of the hallux are both rare and complicated, and these conditions pose great clinical challenge to orthopedists as improper handling can lead to distal tissue necrosis, osteomyelitis, malunion, non-union and even amputation. Due to the lack of soft tissue and bone and the blocking effect caused by un-disconnected soft tissue, it is relatively difficult to achieve effective reduction and fixation of the fracture by traditional methods. Methods: we present a patient, diagnosed with open fracture combined with bone defects of the distal segment of the great toe, who was treated using a self-made adjustable external fixation device by complete debridement, effective closed reduction and continuous adjustment. Complications were registered and clinical outcome was measured at the follow-up periods. Results: The device was simple to make with a low cost and practicality, and this technique resulted in effective maintenance of reduction and progressive compression in the fracture ends. The patient finally obtained optimal fracture healing and satisfactory function of the injured toe at last follow-up. Conclusions: The self-made adjustable external fixation device is reliable in maintaining reduction and fixation, which provides an alternative for the management of open fracture associated with bone absence of the distal phalanx of the hallux.

\section{Introduction}

Open fractures with bone defects of the distal phalanx of the hallux are mainly caused by direct impact, such as crush or chain-saw injury [1]. The latter is relatively less reported in clinical practice [2]. At present, the clinical management of such injuries is primarily based on the classification of open fractures and the degree of bone defects. This involves thorough debridement, reduction and axial fixation (usually by Kirschner wires) that are conducted in one-stage operation [3]. Adjuvant medication is administered to improve blood supply to the distal area postoperatively [3]. However, this is accompanied by the deficiency of soft tissue and bone. The compression to the surrounding soft tissue following Kirschner wire fixation and unbroken soft tissue excess on the distal blood supply, can pose certain challenges to the efficacy of the clinical treatment. These are combined with the difficulties to receive effective closed reduction. In the present study, a case of open fracture with bone defects of the right great toe is described. The patient was treated by self-made external fixation device with a procedure of adjusting the external fixator at different stages. This method aimed to maintain the pressurized state of the fracture ends following the first-stage operation. The surgery was able to treat the wound and fix the fracture ends.

\section{Case}

\section{Basic situation of the patient}

A male patient, who was 47 years old, was delivered to the Emergency Department of Orthopedics in the People's Hospital of the Sihong County (Jiangsu Province), due to pain, bleeding and lack of motion of the right great toe for $2 \mathrm{~h}$. His injury was caused by chain-saw trauma. Physical examination indicated 
that his general condition was optimal and his cardiopulmonary function was normal. The distal segments of the injured toe were mostly disconnected from the proximal side of the toe nail (Fig. 1a), leaving the fracture ends exposed. Only the inner part was connected with the skin and the poor blood supply of the distal segments. X-ray photos demonstrated that the distal hallux phalanx was broken with the separation of fracture ends and bone defects (Fig. 1b). According to the patient's injury history, his symptoms, signs and his auxiliary examination, the distal open fractures of the right great toe with bone deficiency were diagnosed. In the emergency department, the affected limb was elevated and temporarily fixed following disinfection and bandaging of the wound. The incidence and severity of various symptoms, including subsiding, swelling and pain were reduced. Subsequently, the patient was admitted to the department of trauma orthopedics, while actively finishing preoperative preparations for emergency surgery.

\section{Surgery}

Surgery was performed following spinal anesthesia. The patient was placed in the supine position and tourniquets were not used. A pre-operative dose of $2 \mathrm{~g}$ of cefotiam was intravenously infused. Once satisfactory anesthesia was established, the wound was rinsed and general disinfection pads were used in the operative field. Initially, the wound was thoroughly irrigated and debrided without removing the nail. Subsequently, the self-made adjustable gradually-pressurized external fixation device was made and applied to acquire effective reduction and fixation of the fracture. A diagram showing the technique and the device is illustrated in Fig. 2 (patent name: a medical adjustable pressurized external fixation device, patent number: ZL201720348662.7).

The specific intraoperative steps were as follows: Following debridement of the wound without tendon rupture, the fracture ends were adjusted in a parallel location and a $1.5 \mathrm{~mm}$ Kirschner wire was inserted along the axial direction. The wound was sutured and acquired with excellent alignment using $\mathrm{C}$-arm fluoroscopy. The $1.5 \mathrm{~mm}$ Kirschner wire was subsequently inserted from the outside to the inside in the proximal hallux phalanx, taking care to avoid damage to the blood vessels and nerves on both sides of the injured toe. Both ends of the Kirschner wire were folded into the shape of the auricular ring. The rubber pad, button and the self-made twisted crossbar were sequentially inserted through the axial K-wire. The crossbar was established by $1.2 \mathrm{~mm} \mathrm{~K}$-wires with both ends were prefabricated in the shape of an "ear",opposite to the ear ring of the proximal K-wire. A series of $0.8 \mathrm{~mm}$ steel wires were used to penetrate the four auricular rings. The rings were tightened moderately to form an external fixation device, while allowing the monitoring of the blood supply of the distal great toe and their retention in case of compression.

\section{3.postoperative management}

The affected limb was raised following operation and treated with the anti-inflammatory drug cefotiam that was administered at $2 \mathrm{~g}$ intravenously on day 2. Moreover, the anticoagulative drug enoxaparin $(40 \mathrm{mg}$ ) was subcutaneously injected on day 1 . In addition, the vasodilatory drug papaverine $(30 \mathrm{mg})$ was injected intramuscularly on day 3 . The dressing change of the wound was strengthened and the 
device was adjusted to increase pressure once every 3 days. The wound was well healed postoperatively with no infection and necrosis and the fracture ends were effectively fixed by the device with the exception of the slight shortening of the finger (Fig. 3a). The fracture was well-aligned as determined by a postoperative X-ray examination (Fig. 3b). The swelling and the peripheral circulation of the broken toe were closely observed and the patient was discharged from the hospital on the 7th day following operation. After discharge, the dressings were changed as required and the stitches were removed 2 weeks post-surgery without soft-tissue complications, such as infection and necrosis. The steel wires were initially tightened using the auricular structure and the rubber band was subsequently used to maintain a pressurized state by simultaneous observation of the blood supply of the injured toe and by launching of functional exercises of the other toes. The rehabilitation exercises were performed by encouraging the patient to take the initiative to exercise the flexion and the extension of the other toes. Subsequently, he was able to walk with crutches without any weight bearing on the affected toe when both swelling and pain of the affected limb were obviously subsided. The external fixation device and axial K-wire were routinely removed 6 weeks postoperatively. Subsequently, the flexion and extension function of the right great toe was strengthened and weight bearing was gradually increased. Finally, the correction of his limping gait was achieved and the patient returned to his normal daily activities. During follow-up, the complications delayed union, nonunion and re-fracture of the injured toe did not occur, while the appearance of the unhealthy toe was slightly shorter (Fig. 3a). The fracture was well healed without obvious angular and rotating malformations as determined by the 24-month postoperative X-ray exanimation (Fig. 4a). The affected toe was well functioned with fine appearance although it was shortened considerably (Fig. 4b). The recoveries of the extension and flexion of the injured toe were optimal (Fig. 4c,4d).

\section{Discussion}

Toe phalangeal fractures, caused by various trauma mechanisms, are common and account for approximately $3.6-8 \%$ of injuries of the lower extremity [4-5]. Among children, $5-13 \%$ of fractures occur in the foot and of these, fractures of the toes are the most common [6-7]. The majority of fractures of the hallux occur at the distal phalanx and are a result of a direct crushing type of injury. Fractures between the 2 nd and the 5th toe are generally of little consequence and can typically be treated successfully with conservative measures, whereas fractures of the hallux can lead to significant long-term pain and disability [6]. Unfortunately, the hallux is the most common toe that is injured, representing the greatest proportion of all toe phalangeal fractures (38-56\%) [8-9]. Open fractures without the hallux are mostly caused by high-energy injuries, whereas chain-saw damage exhibits low incidence and causes major destruction, which is often accompanied by surrounding soft tissue injury and bone defects. Therefore improper treatment can easily lead to distal tissue necrosis, delayed fracture healing, non-union and other complications, which affect the overall aesthetic appearance and load-bearing function of the toe. Improper treatment may even lead to the possibility of amputation. The treatment of this type of injury is considered a major challenge for trauma orthopedics. The complex structure and flexible function of the foot should be fully considered as a reasonable option combined with physiological anatomy, which is 
directly associated with the recovery of foot function. Kirschner wire fixation, or direct external fixation are inconvenient ways to observe the wound condition and the distal blood flow. The changing of the dressings was generally used on the basis of debridement and suturing in previous studies. Simple axial Kirschner wire fixation can not firmly fix the broken ends and allows poor anti-rotation ability. This may increase the risk of delayed healing and nonunion of the fractures, while cross Kirschner wire fixation avoids anatomical reduction due to the narrow section of the distal phalangeal bone, which increases the difficulties and the time of operation, further damaging the distal blood supply [10-13].

To provide an alternative for these disadvantages, our team designed an adjustable progressive external fixation device on the basis of traditional methods. The applications and the construction of this device are pending patent approval. The injury, often involves the absence of soft tissue and bone and the blocking effect of the unmutilated soft tissue and of the irregular section of the distal phalangeal bone. This inhibits the effective reshaping and placement of the fracture ends. According to previous studies $[1,14]$ it has been shown that fracture healing requires mechanical environment, separation and rotation. Shear stress and other forces are not conducive to the stability and healing of the fractures, which require control. The debridement, reduction, axial K-wire fixation and progressive compression of the external device as well as the contact area and the friction of the fracture ends were effectively increased, reducing the impact of the forces, such as separation, rotation and shear stress. Concomitantly, normal toes can continue to exercise under the broken toe, which is fixed by pressure application. This increases the blood circulation of the broken toe, which is beneficial to fracture healing. This method can potentially reduce the iatrogenic injury of the distal segments under the premise of minimally invasive treatments, including primary debridement, reduction, axial fixation and suturing. Moreover, an auxiliary drug therapy is applied to raise the blood supply of the distal area as much as possible. Following observation of the optimal blood supply of the injured toe, the fracture ends were progressively subjected to axial compression by self-made external device by gradually adjusting the steel wires on both sides. This maintained the compression effect of the fracture ends by the transverse Kirschner wires. The present case indicated that the self-made adjustable external fixation device could achieve progressive axial compression, maintain high stability of fracture ends and lower the interference of the blood supply, which was beneficial to fracture healing and improved the early functional exercise of toes. Finally it increased the recovery of load bearing function, thus rendering it an alternative approach for the complex injury.

The following advantages of the self-made adjustable external fixation device were generally analyzed and concluded: First of all, the device exhibited potent plasticity with reasonable design, simple construction, instant generation in the operation, and high practicability and ease of operation. Moreover, the device exhibited low cost and reduced learning curve. High stability could be efficiently acquired by the process of axial fixation and progressive compression, which could maintain the fracture ends in a stable, gradually-stressed state, thereby promoting fracture healing. In addition, the technique was a relatively minimally invasive operation with low trauma incidence and maximum blood supply protection. Its application can reduce the financial burden of patients as low costs of materials are used and a simple removal of the device can be accomplished in the absence of anesthesia and hospitalization. This 
type of external fixation device exhibits certain shortcomings, mainly manifested in the following aspects: First of all, it is inconvenient to carry and secondly potential nail canal infection may be caused. In addition to this application, transarticular fixation of this method is detrimental to joint movement, notably in the early stage of functional exercises. Therefore, comminuted fractures and fractures involving joint surface are not highly recommended when using this device.

\section{Conclusion}

The self-made adjustable external fixation device of progressive compression is an alternative for the management of open fracture associated with bone absence of the distal phalanx of the hallux. The device is simple to manufacture with a low cost and practicality and is reliable in maintaining reduction and fixation. Moreover, it exhibits lower interference with distal blood supply and it can be further developed for future applications in the clinic.

\section{Declarations}

\section{Ethics approval and consent to participate}

This study was approved by the ethics committee of Sihong county people's hospital. The patients signed a surgical consent form. Informed consent was obtained from the participant in the study.

\section{Consent for publication}

Informed consent was obtained from the participant in the study.

\section{Availability of data and material}

All data generated or analyzed during this study are included in this article.

\section{Competing interests}

The authors declare that they have no competing interests.

\section{Funding}

NO

\section{Authors' contributions}

YGY conceived, designed, performed and analyzed the study, wrote the paper. DKT and PZL designed the study and revised the manuscript. All authors reviewed the results and approved the final version of the manuscript.

\section{Acknowledgements}


Not applicable.

\section{References}

[1] York P J, Wydra F B, Hunt K J. Injuries to the great toe [J]. Current reviews in musculoskeletal medicine, 2017, 10(1): 104-112. doi: 1007/s12178-017-9390-y.

[2] Tucker D J, Jules K T, Raymond F. Nailbed injuries with hallucal phalangeal fractures--evaluation and treatment [J]. Journal of the American Podiatric Medical Association, 1996, 86(4): 170-173. doi: $10.7547 / 87507315-86-4-170$.

[3] Banks A S, Cain T D, Ruch J A. Physeal fractures of the distal phalanx of the hallux [J]. Journal of the American Podiatric Medical Association, 1988, 78(6): 310. doi: 10.7547/87507315-78-6-310.

[4] Shibuya N, Davis M L, Jupiter D C. Epidemiology of foot and ankle fractures in the United States: an analysis of the National Trauma Data Bank (2007 to 2011) [J]. The Journal of Foot and Ankle Surgery, 2014, 53(5): 606- 608. doi: 10.1053/j.jfas.2015.07.016.

[5] Court-Brown C M, Caesar B. Epidemiology of adult fractures: a review [J]. Injury, 2006, 37(8): 691697. doi: 10.1016/j.injury.2006.04.130.

[6] Petnehazy T, Schalamon J, Hartwig C, et al. Fractures of the hallux in children [J]. Foot \& ankle international, 2015, 36(1): 60-63. doi: 1177/1071100714552482.

[7] Kay R M, Tang C W. Pediatric foot fractures: evaluation and treatment [J]. JAAOS-Journal of the American Academy of Orthopaedic Surgeons, 2001, 9(5): 308-319. doi: 5435/00124635-20010900000004.

[8] Van Vliet-Koppert S T, Cakir H, Van Lieshout E M M, et al. Demographics and functional outcome of toe fractures [J]. The Journal of Foot and Ankle Surgery, 2011, 50(3): 307310. doi: 10.1053/j.jfas.2011.02.003.

[9] Eves T B, Oddy M J. Do broken toes need follow-up in the fracture clinic? [J]. The Journal of Foot and Ankle Surgery, 2016, 55(3): 488-491. doi: 10.1053/j.jfas.2016.01.034.

[10] Pinckney L E, Currarino G, Kennedy L A. The stubbed great toe: a cause of occult compound fracture and infection [J]. Radiology, 1981, 138(2): 375-377. doi: 1148/radiology.138.2.7455117.

[11] Nakamura S. Temporary Kirschner wire fixation for a mallet toe of the hallux [J]. Journal of Orthopaedic Science, 2007, 12(2): 190-192. doi: 1007/s00776-006-1106-x.

[12] Wada K, Yui M. Surgical treatment of mallet toe of the hallux with the extension block method: a case report [J]. Foot \& ankle international, 2011, 32(11): 1086-1088. doi: 10.3113/FAl.2011.1086. 
[13] Morris B, Mullen S, Schroeppel P, et al. Open physeal fracture of the distal phalanx of the hallux [J]. The American journal of emergency medicine, 2017, 35(7): 1035. e1-1035. e3. doi: 10.1016/j.ajem.2017.02.015.

[14] Kensinger D R, Guille J T, Horn B D, et al. The stubbed great toe: importance of early recognition and treatment of open fractures of the distal phalanx [J]. Journal of Pediatric Orthopaedics, 2001, 21(1): 3134.x. doi: 10.1097/00004694-200101000-00008.

\section{Figures}

\section{Figure 1}

Preoperative wound and X-ray imaging (a) An open wound was noted in the distal phalanx of the right great toe with the majority of the bone disconnected from the proximal part of the toe nail, leaving the inner part exclusively attached to the skin and resulting in a weak peripheral circulation. (b) AP radiography indicated that the fracture of the distal hallux phalanx was associated with the separation of broken ends and bone defects. 


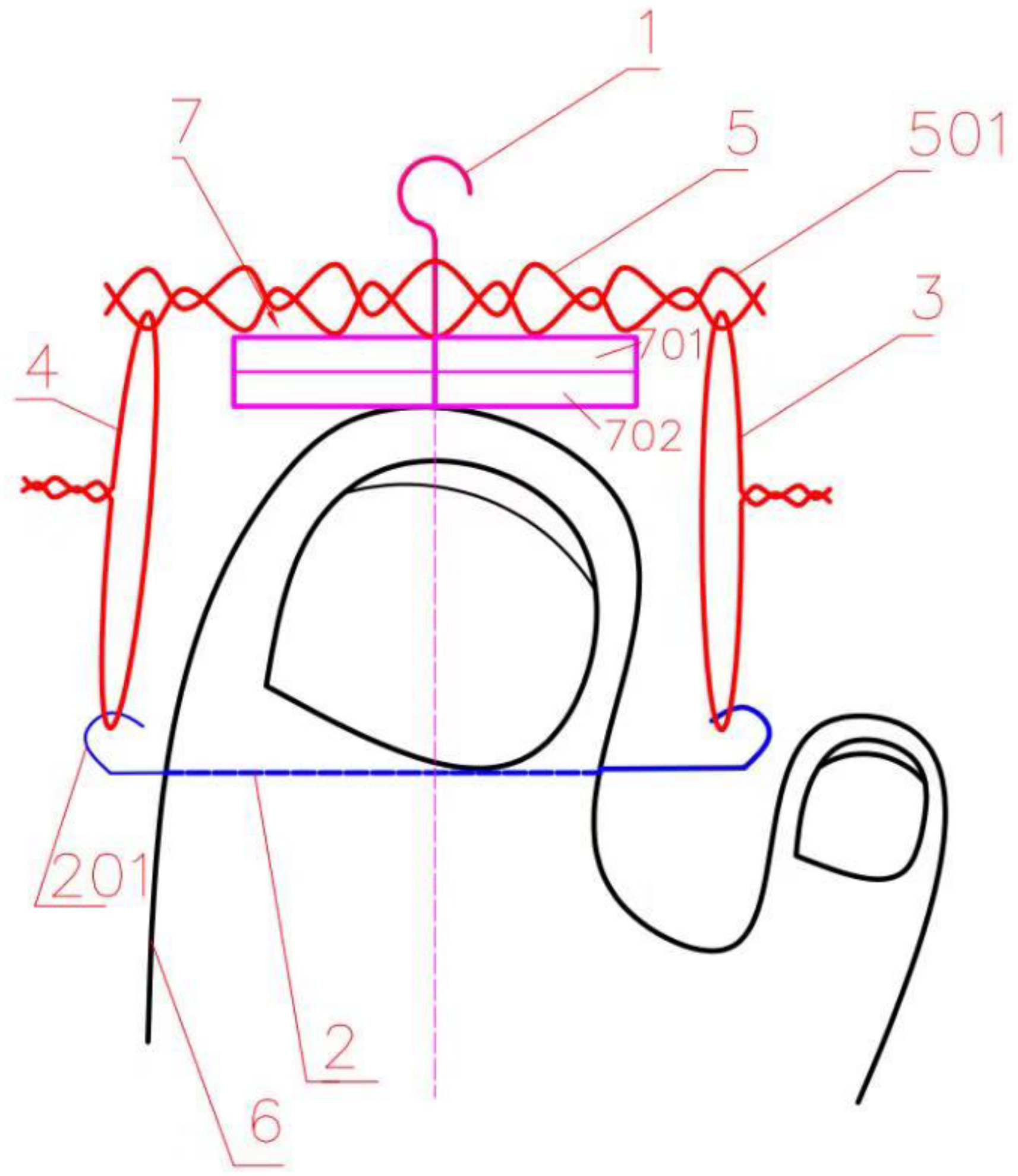

Figure 2

The schematic diagram of the self-made adjustable external fixation device. (1) axial K-wire, (2) transverse K-wire, $(3,4)$ pressurized steel wire, (5) cross bar, (6) the distal hallux phalanx, (201-501) auricular ring, (701) fastener, (702) rubber insert.

2 


\section{Figure 3}

Postoperative wound and X-ray films(day 5) (a) The wound was well healed postoperatively with no infection and necrosis, and the fracture ends were effectively fixed by the device with the exception of the slight shortening of the finger. (b) AP and lateral radiographs of the right hallux following fixation demonstrating that the fracture was well-aligned.

\section{Figure 4}

Postoperative recovery and follow-up results (all photos were from the same patient at 24 months postoperatively). (a) AP and lateral films indicating that the fracture was well healed without nonunion. (b) The affected toe functioned well and had fine appearance and acceptable shortening. (c) The extension function between the uninjured side and the injured side. (d) The flexion function between the unhealthy side and the healthy side. 